\title{
Ensuring the Survivablity of the Trekker Using Drone and RFID Technology
}

\author{
Rathinapriya $\mathrm{V}^{\mathrm{a}, \mathrm{b}, 1}$, Rahul $\mathrm{D}^{\mathrm{b}}$, Rakesh $\mathrm{M}^{\mathrm{b}}$ and Suganthan $\mathrm{P}^{\mathrm{b}}$ \\ a Assistant Professor \\ ${ }^{\mathrm{b}}$ Computer Science and Engineering, Easwari Engineering College, Chennai, India
}

\begin{abstract}
Amature trekkers venturing into the forest often tend to lose their path and find it difficult to return back to their origin or destination. It is important for the rescuers and volunteers to promptly find the trekker in order to increase the probability of survival. Imparting confidence and providing proper guidance to the trekker is also an important part in search and rescue missions. Drones can be used to locate the trekker promptly using RFID technology and global positioning system (GPS) and provide communication systems between the trekker and the rescue team using LoRa.
\end{abstract}

Keywords. Drone, RFID Technology, Global Positioning System (GPS), LoRa.

\section{Introduction}

Mass migration towards cities has happened in recent years, yet people love the forest and its surroundings. This has led to rise in forest based tourism all around the world. Trekking is the most exciting and preferred activity. Trekking also has dangers associated with it. One of the basic dangers is losing the path and being unable to reach the destination or return back to the starting point. For ammeture trekkers the situation can turn dangerous without any guidance. With every moving minute, the probability of survival decreases. Due to the anxiety and fear the victim might make wrong decisions which may lead the trekker to more grievous situations. In this modern age, the world is betting on drones for tackling all kinds of situations, from national security to survey to delivering products. Due to the aerial navigation of drones, it can reach any point promptly. Such promptness can be used to search through the forest and locate the missing person. The aerial vehicle can use advanced technology of radio frequency identification to accurately locate the person. Global navigation systems have grown in large to help the aerial vehicle to navigate and to send the location of the missing person to the rescue teams. The aerial vehicles can be equipped with modern long range communication devices for transfer of data and to establish communication.

\footnotetext{
${ }^{1}$ Rathinapriya V, Assistant Professor, Department of Computer Science and Engineering, Easwari Engineering College, Chennai; E-mail: rathinapriya.v@eec.srmrmp.edu.in
} 


\section{Literature sources}

Rohei et al [1] proposed a system in which the RFID system is used to detect the humans in the indoor environment. The RFID proposed in this system is operated through IOT, where all the data of the RFID is stored in the internet. This system also proposes the use of RFID embedded cloth tags or even bracelets, keychains etc., which can be used to track people using this technology. It is highly inefficient since it can be used only indoors and due to using passive RFID the range is less.

Buffi et al. [2] proposed a system in which they use Multilateration algorithm to locate the distance of the tag from reader. The presence of the tag is found by the backscattered signal from the tag which is sensed by the RFID reader. It also uses SAR based localisation algorithm to investigate uncertainty in measurement related to reader antenna trajectory and UHF-RFID Reader which improves the measurement accuracy of the system. It has a major issue that it requires communication lines between the distant receivers and transmitters.

Albanese et al. [3] proposed a system in which the use autonomous drones with high penetration signal to find mobile phones which intern can be used to find people. The main disadvantage is that it works only when the mobile phone is present in undamaged condition and it requires high resolution cameras in order to find the victim of locating the phone.

Rohman et al. [4] proposed a system to use multiple sensors like UWB radar, microphone array, camera and RFID reader assembled in a drone to detect and geo localise the survivor in post disaster environments. This system is interconnected with a main server and a mission ground control, when the location of an survivor is detected in the disaster environment by the drone, the real time location data is shared with the main server which is monitored by the rescue team. To improve accuracy of this system, sensors with high specialisation are required which makes operational cost higher. G. Castellano and etal [5] proposed a system with Drones that uses UAV equipped with high resolution camera and TinyYOLOv3 for object detection to automatically detect victims in Rescue scene.The dataset designed for search and rescue operation is small which can be used only for testing and evaluation purpose. To implement this system in real time, larger dataset will be required. High resolution cameras increase the cost of the project.

Jhunjhunwala et al. [6] proposed a system that discusses the application of RFID equipped in the drone for product scanning and stock management in a warehouse. The drone uses sampling based algorithm to choose between multiple paths. The stability of the drone during vibration test need to be improved. Li et al. [7] proposed a system with a RFID based tracking system for I constrained indoor environment. Particle swarm optimisation algorithm is used by the system to determine the initial position, there after a dynamic correction method for trajectory prediction is used for Continuous tracking of the object. RFID technology based localisation approaches can be affected greatly by the random moving objects in the field due to signal fluctuation and multi-path effect, which in turn can reduce the accuracy. It also limits the further development of RFID-based indoor positioning technology. 


\section{Existing Technology}

\subsection{Radio frequency identification system}

Radio frequency tags are used to track humans in an indoor environment, with the help of multiple radio frequency identification scanners. [1]

\subsection{High signal penetration with computer vision}

High signal penetration is used to find the mobile phones that are present near the victims. Then the computer vision is used to identify the human. [3]

\subsection{High resolution cameras}

High resolution cameras along with TinyYOLO are used for object detection and then to detect the humans.

\section{Proposed System}

The proposed system aims to use a quadcopter [8], which can fly in the predefined path on its own using a global positioning system, magnetic compass, barometer and accelerometer. The quadcopter also consists of the radio frequency identification (RFID) scanner to scan the radio frequency identification (RFID) tag which will be present with the trekker, thereby accurate location of the trekker can be found promptly and accurately [9-11]. On finding the trekker the coordinates of the location is obtained by the quadcopter using a global positioning system. The coordinates are transmitted back to the base station and the rescue team using LoRa, a long range communication device [12]. The drone is also equipped with communication devices namely speakers and microphone to enable dual way communication between the trekker, rescue team and the base station $[13,14]$.

\section{Architecture}

\subsection{Drone}

The Drone is provided with four carbon blades of dimension $10 \mathrm{X} 4.5$ to provide greater stability. These four wings are mounted on the four $1000 \mathrm{KV}$ motors respectively, which can provide a 2000 RPM to 6000 RPM. The speed of these four motors are controlled by four electronic speed controllers respectively, which are rated at 30A. Electronic speed controllers are controlled by the arduino based controller which is equipped with a global positioning system to provide the current location and move to upcoming coordinates, a magnetic compass to provide the sense of moving direction to quadcopter, a barometer to provide the quadcopter with current altitude so as to maintain the height, an accelerometer to provide the sense of acceleration applied to the quadcopter and a gyroscope to determine the changes in position and movement. The quadcopter controller and the electronic speed controllers are powered by 3 lithium ion batteries each with 2500 $\mathrm{mAh}$, which are lighter in weight and has more capacity. 


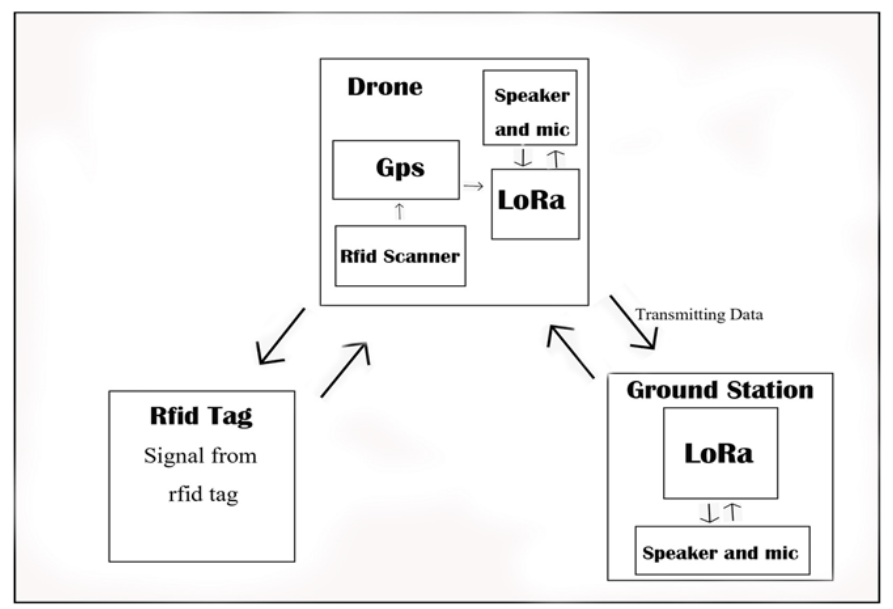

Figure 1. Architecture of the Proposed System

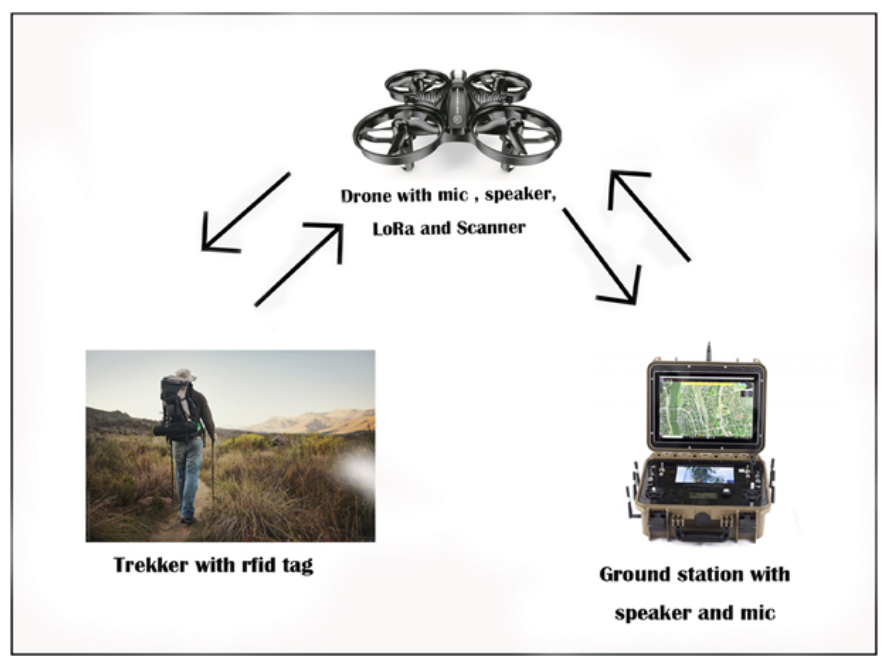

Figure 2. Proposed System

\subsection{Location Identification System}

The quadcopter will have the radio frequency identification (RFID) scanner, which will be programmed with an arduino to scan for active radio frequency identification tag. Once the scanner receives a signal back from the RFID tag, the GPS coordinates of the 


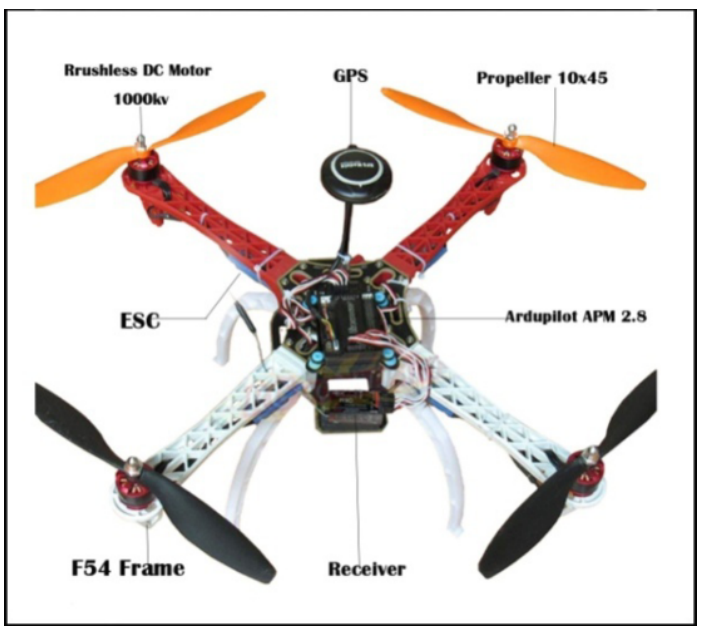

Figure 3. Drone

main loop \{

- Get raw GPS, gyroscope, accelerometer, barometer and magnetic compass data

- Update estimate of location, pitch, roll and yaw

- PID controllers: Calculate a desired gyro velocity for each angle based on the difference between the current quadcopter pitch/roll/yaw and the desired pitch/roll/yaw (i.e. gyro_pitch_desired = (desired_pitch -

actual_pitch) * Kp) Using the calculated desired angular rotation rates and the actual angular rotation rates, use PID controllers to produce an output to mix with the throttle for the motors.

- Calculate motor PWM using the output from the pitch, roll and yaw PID controllers.

- Send PWM pulses to ESCs.

-

\}

Figure 4. Pseudocode

same location are obtained and the same is transferred back to the base station and rescue team using a long range communication system called LoRa. The drone stays in the same location until the help arrives.

\subsection{Communication System}

Establishing communication is an important part of ensuring survivability of the trekker. Therefore quadcopter is equipped with speakers and mic to establish dual way communication systems. The sounds can be transferred via LoRa to the base station and the rescue team. 


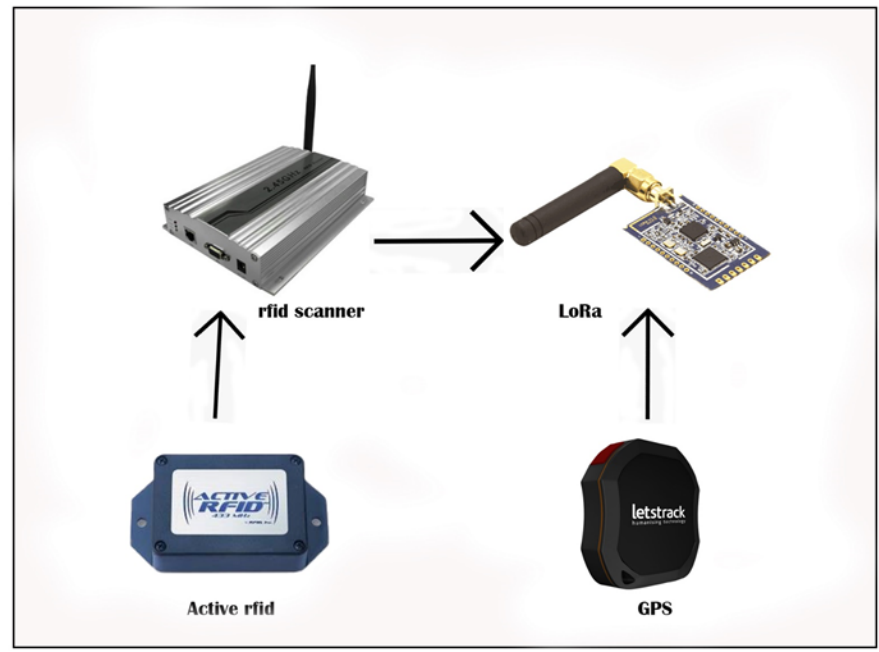

Figure 5. Location Identification System

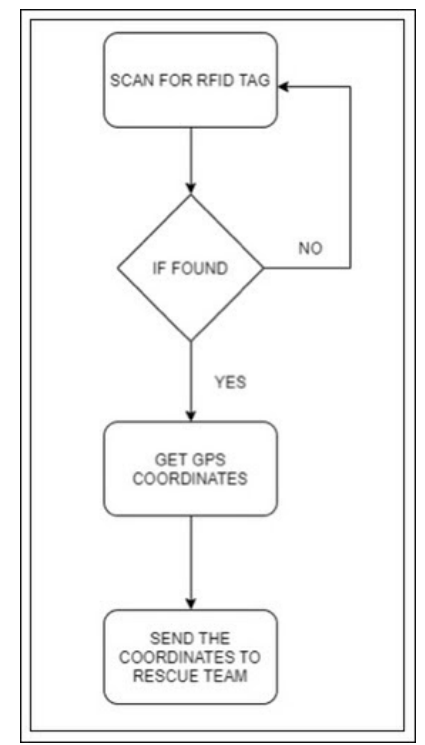

Figure 6. Location Identification System (Flow diagram)

\section{Result}

The quadcopter moves on its predefined path when deployed and scans for the radio frequency identification tag. Once spotted the quadcopter gets the GPS location and relays it back to the base station. The interaction between the base station and the missing trekker can also be done. 


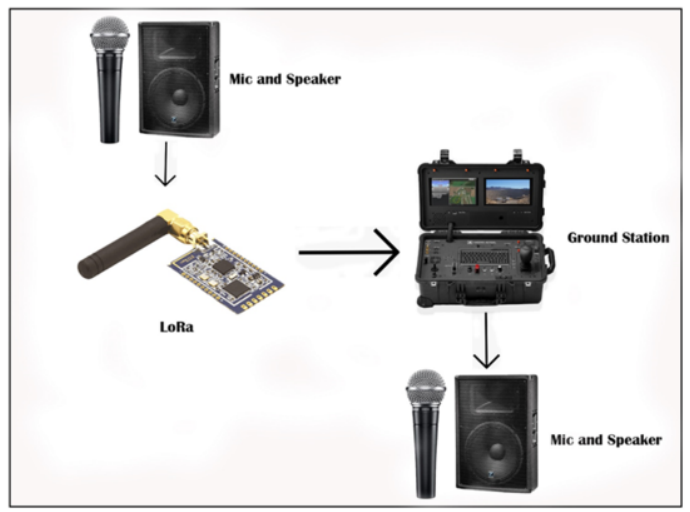

Figure 7. Dual way communication System

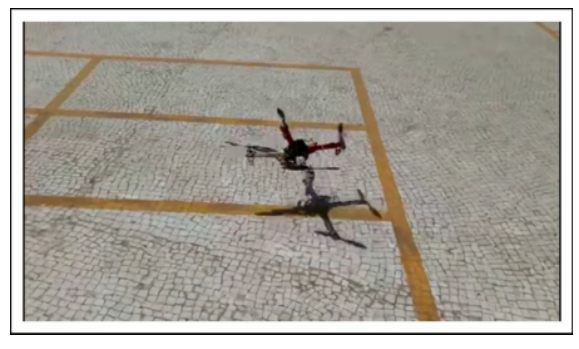

Figure 8. Drone- Ready to Test.

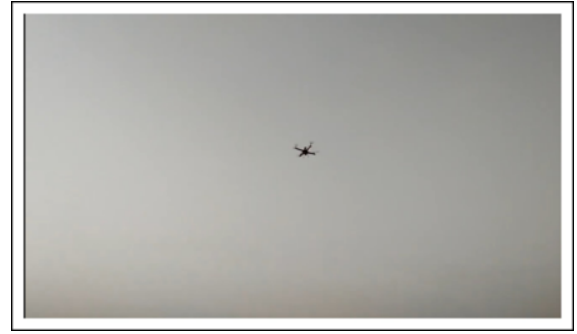

Figure 9. Drone - Testing.

Figure 8 shows the drone that is ready to fly on the requirement. It can be deployed at anyplace. The search co-ordinates need to be fed via mission planner. Figure 9 shows the drone that is flying in via specified co-ordinates in search of the missing person. Figure 10 shows the path that is covered by the drone. Figure 11 shows the page that display the the co-ordinaries of the missing person found by the drone. Mic button can be used to talk to the missing person via drones communication system.

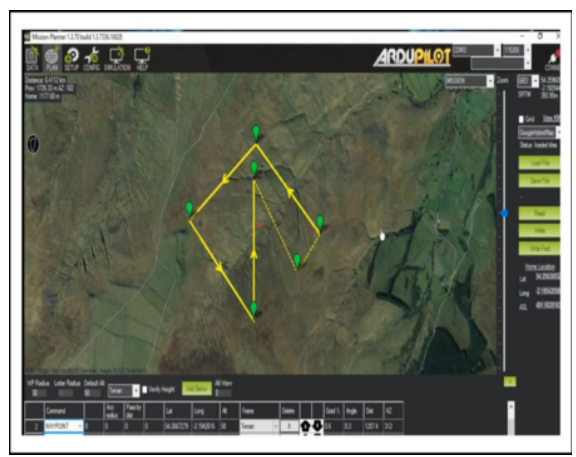

Figure 10. Ground Console.

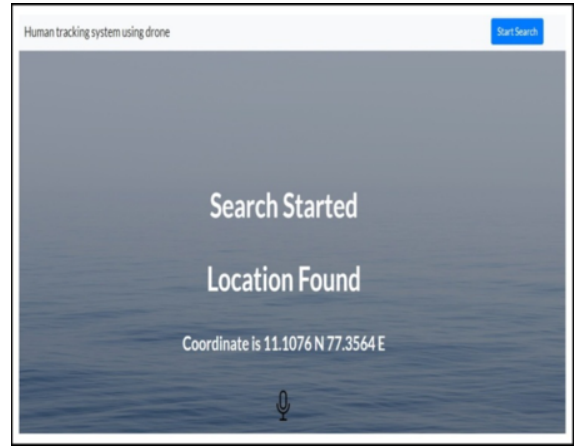

Figure 11. Output. 


\section{Conclusion And Future Work}

Thus the quadcopter system created can be promptly deployed for the identification of the missing person and to provide support through voice communication, thereby increasing the survivability of the person. The quadcopter operation does not require any specialisation. In future the quadcopter can be programmed to use cameras and artificial intelligence technology to pick up the trails of the missing person from the last known location thereby reducing the time to find the missing person. Also the quadcopter can be allowed to get the last known location of the person, thereby allowing itself to program in such a way to find the most probable path the person might have chosen. Introducing a camera in a quadcopter will also allow the rescue team to identify situations in time of uncertainty.

\section{References}

[1] Rohei MS, Salwana E, Shah NB, Kakar AS. Design and testing of an epidermal rfid mechanism in a smart indoor human tracking system. IEEE Sensors Journal. 2020 Nov 5;21(4):5476-86.

[2] Buffi A, Tellini B. Measuring UHF-RFID tag position via unmanned aerial vehicle in outdoor scenario. In2018 IEEE 4th International Forum on Research and Technology for Society and Industry (RTSI) 2018 Sep 10 (pp. 1-6). IEEE.

[3] Albanese A, Sciancalepore V, Costa-Pérez X. SARDO: An automated search-and-rescue drone-based solution for victims localization. IEEE Transactions on Mobile Computing. 2020 Mar 12:1-13.

[4] Rohman BP, Andra MB, Putra HF, Fandiantoro DH, Nishimoto M. Multisensory surveillance drone for survivor detection and geolocalization in complex post-disaster environment. In IGARSS 2019-2019 IEEE International Geoscience and Remote Sensing Symposium 2019 Jul 28 (pp. 9368-9371). IEEE.

[5] Castellano G, Castiello C, Mencar C, Vessio G. Preliminary Evaluation of TinyYOLO on a New Dataset for Search-and-Rescue with Drones. In 2020 7th International Conference on Soft Computing \& Machine Intelligence (ISCMI) 2020 Nov 14 (pp. 163-166). IEEE.

[6] Jhunjhunwala P, Shriya M, Rufus E. Development of hardware based inventory management system using uav and rfid. In 2019 International Conference on Vision Towards Emerging Trends in Communication and Networking (ViTECoN) 2019 Mar 30 (pp. 1-5). IEEE.

[7] Li J, Feng G, Wei W, Luo C, Cheng L, Wang H, Song H, Ming Z. PSOTrack: A RFID-based system for random moving objects tracking in unconstrained indoor environment. IEEE Internet of Things Journal. 2018 Jan 22;5(6):4632-41.

[8] Kwak J, Sung Y. Autonomous UAV flight control for GPS-based navigation. IEEE Access. 2018 Jul 10;6:37947-55.

[9] Hutabarat DP, Hendry H, Pranoto JA, Kurniawan A. Human tracking in certain indoor and outdoor area by combining the use of RFID and GPS. In 2016 IEEE Asia Pacific Conference on Wireless and Mobile (APWiMob) 2016 Sep 13 (pp. 59-62). IEEE.

[10] Subedi S, Pauls E, Zhang YD. Accurate localization and tracking of a passive RFID reader based on RSSI measurements. IEEE Journal of Radio Frequency Identification. 2017 Oct 23;1(2):144-54.

[11] Çavur M, Demir E. Real-time localization methodology with RFID technology in closed area. In 2017 25th Signal Processing and Communications Applications Conference (SIU) 2017 May 15 (pp. 1-4). IEEE.

[12] Alshareef HN, Grigoras D. Multi-service cloud of drones for multi-purpose applications. In 2017 16th International Symposium on Parallel and Distributed Computing (ISPDC) 2017 Jul 3 (pp. 165-174). IEEE.

[13] Olejnik A, Kiszkowiak Ł, Rogólski R, Chmaj G, Radomski M, Majcher M, Omen Ł. Precise remote sensing using unmanned helicopter. In 2019 IEEE 5th International Workshop on Metrology for AeroSpace (MetroAeroSpace) 2019 Jun 19 (pp. 544-548). IEEE.

[14] Alotaibi ET, Alqefari SS, Koubaa A. Lsar: Multi-uav collaboration for search and rescue missions. IEEE Access. 2019 Apr 22;7:55817-32. 